\title{
Estilos cerebrales de pensamiento vinculados al comportamiento del consumidor en estudiantes universitariosi
}

\section{Brain thinking styles related to the consumer behaviour in university students}

\author{
Manuel Pedro Torres Lajo ${ }^{2}$ \\ Technische Universität München. Alemania \\ manuel.torres-lajo@tum.de \\ https://orcid.org/0000-0003-3085-1394
}

Aceptado: 07/08/2019

\begin{abstract}
RESUMEN
El presente artículo tiene como objetivo establecer la correlación existente entre los Estilos Cerebrales de Pensamiento y el Comportamiento del Consumidor en una muestra de tipo probabilístico compuesta por 318 estudiantes de la facultad de economía de una universidad pública de la ciudad de Lima, Perú. A estos alumnos se les aplicaron dos instrumentos con niveles consistentes de validez y confiabilidad: el Inventario de Estilos Cerebrales de Pensamiento de Carlos Alberto Jiménez y el Inventario de Comportamiento del Consumidor de Sproles y Kendall, el cual fue adaptado por el autor de este artículo para efectos de la investigación. Los resultados obtenidos revelan la influencia que tienen los Estilos Cerebrales de Pensamiento sobre el Comportamiento del Consumidor en la muestra estudiada, lo cual se refleja tanto en el Consumidor más emocional como en el consumidor más racional. En ambos factores, se aprecian los efectos de la emoción en el comportamiento del consumidor, porque aún en la racionalidad, los sectores emocionales del cerebro
\end{abstract}

(C) Los autores. Este artículo es publicado por Pensamiento Crítico de la Facultad de Ciencias Económicas, Universidad Nacional Mayor de San Marcos. Este es un artículo de acceso abierto, distribuido bajo los términos de la licencia Creative Commons Atribucion - No Comercia_Compartir Igual 4.0 Internacional. (http:// creativecommons.org/licenses/by-nc-sa/4.0/) que permite el uso no comercial, distribución y reproducción en cualquier medio, siempre que la obra original sea debidamente citada. 
influyen en el comportamiento del consumidor.

Palabras clave: Estilos Cerebrales de Pensamiento; Comportamiento del Consumidor; Economía del Comportamiento; Neuroeconomía; Decisión de Compra.

JEL: D91, D87, D12, M31.

\section{ABSTRACT}

This article has as main objective to establish the existent correlation between the Brain Thinking Styles and Consumer Behaviour in a probabilistic sample of 318 students from the economics faculty of a public university in Lima, Peru. Two instruments, with consistent levels of validity and reliability, were applied to these students: the Inventory of Brain Thinking Styles of Carlos Alberto Jiménez, and the Consumer Style Inventory of Sproles and Kendall, which the author of this article adapted for research purposes. The results show the influence that the Brain Thinking Styles have on the Consumer Behaviour in the studied sample. This is reflected in both the behaviour of the more emotional consumer and the more rational consumer. In both factors, it is possible to appreciate the effects that emotions have on consumer behaviour, because, even in rationality, the emotional areas of the brain have an influence.

Key words: Brain Thinking Styles; Consumer Behaviour; Behavioural Economics; Neuroeconomics; Purchase Decision.

JEL: D91, D87, D12, M31. 


\section{Introducción: la Economía del Comportamiento}

Todos los seres humanos tienen necesidades de diverso orden, que pueden o no ser fundamentales para la vida pero que, en todos los casos, requieren ser resueltas para que se garantice el estado de bienestar de los sujetos. En este proceso, puede ser necesaria la adquisición de bienes o servicios que pueden ser producidos por personas, de manera individual o como empresas, que buscan satisfacer las necesidades de sus clientes de manera que puedan fidelizarlos y mantenerlos como tales, a través del tiempo. Es importante señalar que, las empresas durante mucho tiempo han dedicado sus mayores esfuerzos a las tareas de producción a fin de lograr mayores beneficios de orden económico. La ecuación siempre fue sencilla: a mayor producción, mayores ganancias.

Pero los tiempos cambiaron. Las condiciones económicas, sociales, tecnológicas, etc., han modificado sustantivamente las formas de vida, el sistema de relaciones de las personas, incluidos sus comportamientos. Por esta razón es que, en la actualidad, ya no es suficiente la producción para obtener ganancias. Hoy en día se requiere que las empresas conozcan mejor a sus consumidores habituales y potenciales, pues a partir de ese conocimiento pueden diseñar y producir bienes y servicios acordes a sus necesidades, logrando, de esta manera, aumentar significativamente los beneficios que se obtendrían en la forma tradicional.

En los nuevos tiempos y gracias al uso de las nuevas tecnologías, el consumidor está mejor informado y ya no solo se limita a recibir la información sobre un producto determinado proporcionada por la empresa fabricante. Los consumidores actuales buscan, además, información en diversas fuentes como organizaciones de consumidores (como la organización alemana Stiftung Warentest que mensualmente publica en su revista comparaciones de diferentes productos), foros en páginas web donde se debate sobre las bondades de un bien o servicio, cuentas de redes sociales de influentes (mejor conocidos por su término en inglés, influencers) que recomiendan y/o prueban productos, etc. Además, aprecian la posibilidad de interactuar directamente con la empresa en tiempo real a través de las redes sociales para absolver dudas, reclamar o buscar soluciones a los problemas que puedan presentar. Por ello, conocer al consumidor se torna crucial para una empresa. 
Aunque un consumidor decida buscar información antes de tomar una decisión, no significa que siempre vaya a tomar la decisión más racional. Así, la racionalidad en economía no necesariamente es la que proponen las teorías económicas más difundidas, las cuales indican que el ser humano evalúa racionalmente antes de decidir. Luego de la crisis financiera global del 2008, se buscaron nuevos campos de estudio que expliquen mejor cómo funciona la economía en situaciones cotidianas (decisiones de compras, comportamiento de mercados financieros, etc.), en donde no necesariamente existen decisiones racionales. Uno de esos campos de estudio es la economía del comportamiento.

Este campo de estudio tiene sus orígenes en la década de los setenta, con el trabajo sobre la Teoría Prospectiva (Prospect Theory) de Daniel Kahneman y Amos Tversky (1979), la cual muestra que los seres humanos no somos tan racionales al momento de tomar decisiones de riesgo en materia económica, como presuponía la Teoría de la Utilidad clásica. Este trabajo le valió a Kahneman el Premio Nobel de Economía en el año 2002. Los trabajos de economistas y psicólogos como Richard Thaler (Premio Nobel de Economía 2017) y Dan Ariely han permitido profundizar más este campo de estudio y ampliar las posibilidades de aplicación del mismo. Por ejemplo, Ariely (2008) muestra en un experimento como la ubicación de los precios en un anuncio de suscripciones a una revista induce al potencial suscriptor a tomar la opción más cara, aun cuando no necesariamente se trate de la mejor alternativa.

En los últimos tiempos se han integrado a este campo de estudios las neurociencias, permitiendo así el estudio de las bases neuroanatómicas y neurofisiológicas ligadas al comportamiento social, y también al económico. A pesar de los limitados conocimientos que aún se tienen sobre el cerebro humano, sabemos que todas las decisiones humanas (voluntarias o no) se toman a nivel cerebral y que cada una activa una zona diferente del cerebro. También podemos afirmar que no todos los seres humanos piensan de la misma forma, lo cual se puede ver reflejado en las reacciones de un sujeto ante una determinada situación. Por ejemplo, si en una tienda se anuncia una rebaja significativa de última hora, es altamente posible que un grupo de personas acuda presurosamente a comprar ese artículo (aunque no lo necesite), mientras que otro grupo ignorará el anuncio (porque puede que no lo necesite o no le interese). Según explican las neurociencias, 
la conexión entre los neurocircuitos corticales (los más racionales) y los centros emocionales del sistema límbico (como la amígdala, la ínsula, etc.) es constante y permanente en ambos sentidos.

El uso de escáneres cerebrales para investigaciones en el campo de la neuroeconomía ha permitido mostrar que diferentes áreas cerebrales se activan ante los diferentes estímulos a los que somos sometidos al estar frente a un producto, una publicidad, o similares. Siguiendo con el ejemplo anterior, el simple anuncio de una rebaja activará áreas vinculadas a la emoción en varios compradores, y en muchos casos la emoción se traducirá en compra. Pero en algunos de ellos, los efectos de la emoción se verán desactivados por las áreas más racionales, que mandan información relacionada, por ejemplo, al precio, el cual puede estar fuera del presupuesto personal del comprador. Este tipo de conocimiento otorga un amplio espectro de posibilidades a una empresa, desde establecer un precio adecuado, diseñar un empaque o toda una campaña publicitaria, por citar algunos ejemplos.

\subsection{Problemas de investigación}

1. ¿Qué influencia tienen los estilos cerebrales de pensamiento en el comportamiento del consumidor de los estudiantes de la Facultad de Economía de una Universidad Pública de Lima?

2. ¿Qué relaciones existen entre los Estilos cerebrales de pensamiento y el comportamiento del consumidor más emocional en los estudiantes de la Facultad de Economía de una Universidad Pública de Lima?

3. ¿Qué relaciones existen entre los Estilos cerebrales de pensamiento y el comportamiento del consumidor más racional en los estudiantes de la Facultad de Economía de una Universidad Pública de Lima?

4. ¿De qué manera los estilos cerebrales de pensamiento predicen la presencia del comportamiento del consumidor más emocional en los estudiantes de la Facultad de Economía de una Universidad Pública de Lima? 
5. ¿De qué manera los estilos cerebrales de pensamiento predicen la presencia del comportamiento del consumidor más racional en los estudiantes de la Facultad de Economía de una Universidad Pública de Lima?

\subsection{Objetivos}

1. Determinar la influencia que tienen los estilos cerebrales de pensamiento en el comportamiento del consumidor de los estudiantes de la Facultad de Economía de una Universidad Pública de Lima.

2. Establecer las relaciones que existen entre los Estilos cerebrales de pensamiento y el comportamiento del consumidor más emocional en los estudiantes de la Facultad de Economía de una Universidad Pública de Lima.

3. Identificar las relaciones que entre los Estilos cerebrales de pensamiento y el comportamiento del consumidor más racional en los estudiantes de la Facultad de Economía de una Universidad Pública de Lima.

4. Determinar si los estilos cerebrales de pensamiento predicen la presencia del comportamiento del consumidor más emocional en los estudiantes de la Facultad de Economía de una Universidad Pública de Lima.

5. Establecer si los estilos cerebrales de pensamiento predicen la presencia del comportamiento del consumidor más racional en los estudiantes de la Facultad de Economía de una Universidad Pública de Lima.

\subsection{Hipótesis}

1. Los estilos cerebrales de pensamiento Influyen sobre el comportamiento del consumidor en los estudiantes de la Facultad de Economía de una Universidad Pública de Lima.

2. Existen relaciones significativas entre los Estilos cerebrales de pensamiento y el comportamiento del consumidor más emocional en los estudiantes de la Facultad de Economía de una Universidad Pública de Lima. 
3. Existen relaciones significativas entre los Estilos cerebrales de pensamiento y el comportamiento del consumidor más racional en los estudiantes de la Facultad de Economía de una Universidad Pública de Lima.

4. Los estilos cerebrales de pensamiento predicen la presencia del comportamiento del consumidor más emocional en los estudiantes de la Facultad de Economía de una Universidad Pública de Lima.

5. Los estilos cerebrales de pensamiento predicen la presencia del comportamiento del consumidor más racional en los estudiantes de la Facultad de Economía de una Universidad Pública de Lima.

\section{Marco Teórico}

\subsection{Los Estilos Cerebrales de Pensamiento}

Siguiendo las propuestas de las neurociencias, es muy importante señalar los aportes contenidos en el modelo de estilos cerebrales de pensamiento de Ned Herrmann, conocido como el Modelo del Cerebro Total, el mismo que permite analizar las preferencias de pensamiento personales y de la organización. Hablamos de estilos de pensamiento porque, tal como se ha mencionado anteriormente, todos los seres humanos piensan diferente. Conocer esos estilos es clave para entender cómo procesan y asimilan la información; cómo deciden y cómo solucionan problemas las personas. Los estilos son dinámicos y pueden cambiar como consecuencia de las experiencias vividas; no obstante, tener claridad sobre los estilos dominantes en un momento dado es importante pues permite establecer un determinado tipo de consumidor.

Según el Modelo de Herrmann, existen cuatro estilos cerebrales de pensamiento, y cada uno de ellos se ubica en una zona determinada del cerebro, a la que se denomina cuadrante. Cada estilo es diferente, pues procesan la información de manera diferenciada; y pese a que pueden funcionar de manera individual, también pueden hacerlo de manera combinada, secuencial o simultánea en los diferentes procesos cerebrales. 


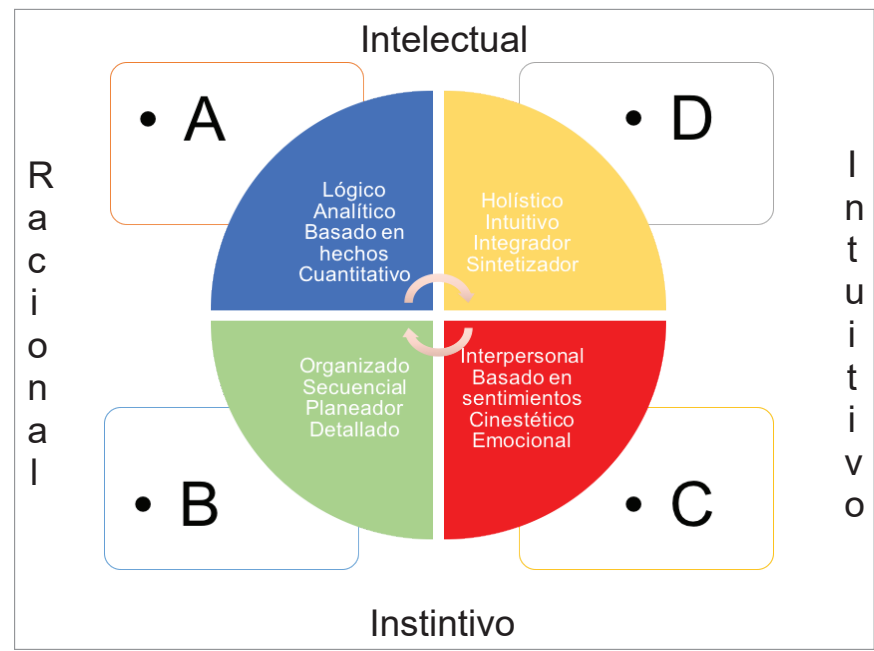

Figura 1. Los cuadrantes cerebrales del Modelo del Cerebro Total de Herrmann

Fuente: Herrmann International (2009)

De acuerdo a la Figura 1, podemos apreciar que el cuadrante A es el lóbulo superior izquierdo; el cuadrante B, el lóbulo inferior izquierdo; el cuadrante $\mathrm{C}$, el lóbulo inferior derecho; y el cuadrante C, el lóbulo superior derecho. De acuerdo al sitio web de Herrmann International (s.f.):

El Cuadrante A tiene un estilo de pensamiento lógico, cuantitativo, analítico, crítico, matemático y basado en hechos concretos.

El Cuadrante B prefiere una estructura de pensamiento secuencial, controlado, detallado, organizado y conservador.

El Cuadrante $\mathrm{C}$ posee un estilo de pensamiento emocional, musical, humanístico, expresivo, sensorial, simbólico y espiritual.

El Cuadrante D destaca por un estilo de pensamiento metafórico, integrador, visual, sintetizador, conceptual, holístico, integrador, creativo, artístico y espacial.

En base a estas definiciones, Pico (2016) asigna un nombre para cada cuadrante: el A es conocido como "Experto"; el B, "Organizador"; el C, "Comunicador"; y el D, "Estratega”. Se usarán estos nombres, así como 
la letra correspondiente a cada estilo, en los análisis de las secciones siguientes.

\subsection{Comportamiento del Consumidor}

Existen diversas definiciones de Comportamiento del Consumidor. Schiffman y Kanuk (2010) lo definen como "el comportamiento que los consumidores exhiben al buscar, comprar, utilizar, evaluar y desechar productos y servicios que ellos esperan que satisfagan sus necesidades". Hoyeret al (2018) afirman que el comportamiento del consumidor "refleja la totalidad de las decisiones de los consumidores a lo largo del tiempo respecto a la adquisición, consumo y disposición de bienes, servicios, actividades, experiencias, personas e ideas".

Todos los consumidores finales forman el mercado de consumidores. Cada mercado se comporta de manera diferente, dependiendo de diversos factores. Kotler y Armstrong (2012) plantean una división de cuatro factores: Culturales, Sociales, Personales y Psicológicos. Estos factores influyen sobre las decisiones que toma el consumidor. Para Sproles y Kendall (1986), es importante identificar los estilos del consumidor para poder perfilar un estilo para cada consumidor, lo cual permitirá educarlos sobre sus características de toma de decisiones y aconsejarlos en la administración de su dinero. Estos autores elaboraron en 1986 el Cuestionario de Estilos del Consumidor (CSI, por sus siglas en inglés), en el que clasificaron a los consumidores en diferentes grupos dependiendo de sus estilos de toma de decisión de compra (Sproles y Kendall, 1986; Sivakumar y Kamaraj, 2014):

i. Perfeccionista: es un consumidor altamente consciente de lo que busca, por lo que revisa cuidadosamente las características de los productos hasta encontrar uno que sea, para él o ella, el de mejor calidad.

ii. Consciente de marca: es un consumidor que prefiere las marcas más conocidas, aun cuando estas puedan resultar más caras que otras opciones en el mercado.

iii. Consciente de la novedad: este tipo de consumidores gustan de las últimas novedades en los productos y disfrutan buscándolos y comprándolos. 
iv. Recreacional: estos consumidores compran por el simple placer de hacerlo.

v. Consciente del precio: es un consumidor que busca la mejor relación calidad-precio, por lo que evalúa el precio del producto antes de adquirirlo. Tiene también inclinación a comprar durante los periodos de descuentos.

vi. Impulsivo: como su mismo nombre lo indica, son consumidores que compran con la emoción del momento, sin reparar en los costos que ello le puede significar.

vii. Confundido por las opciones: son consumidores que se ven abrumados por los diferentes productos, las diferentes tiendas y en general cuando existe una amplia variedad de opciones de dónde escoger.

viii.Leal a una marca: estos consumidores tienen claras sus preferencias por determinadas marcas, por lo que las adquieren siempre. Es decir, es un hábito en ellos adquirirlas.

Para efectos del presente artículo, se ha agrupado a estos grupos de estilos del consumidor en Factor 1 (F1), el cual está compuesto por los consumidores Recreacionales, Conscientes de la Novedad (Moda), Conscientes de Marca y Leales a una Marca; y en Factor 2 (F2), compuesto por los consumidores Perfeccionistas, Conscientes del Precio y los Confundidos por las Opciones. En adelante denominaremos al Factor 1 como Factor o Consumidor más Emocional. Por su parte, el Factor 2 se denominará como Factor o Consumidor más Racional.

\section{Metodología}

\subsection{Método}

Se utilizó el método descriptivo (Sánchez y Reyes, 2006) pues este describe las situaciones y eventos, y también mide diversos aspectos del fenómeno a investigar. El método descriptivo evalúa de manera independiente las variables y deja abierta la posibilidad de realizar predicciones si acaso se utilizan los estadísticos correctos. A este tipo de investigaciones se les conoce como Ex Post Facto (Kerlinger y Lee, 2002) en tanto el investiga- 
dor no tiene manejo directo sobre las variables independientes porque ya ocurrieron sus manifestaciones.

\subsection{Diseño de investigación}

Siguiendo lo propuesto por Hernández, Fernández y Baptista (2014), el diseño a utilizar, en términos generales, es el diseño no experimental y en términos específicos es un diseño correlacional causal.

Los diseños correlacionales-causales pueden limitarse a establecer relaciones entre variables sin precisar sentido de causalidad o pretender analizar relaciones de causalidad. Cuando se limitan a relaciones no causales, se fundamentan en planteamientos e hipótesis correlaciónales, del mismo modo, cuando buscan evaluar vinculaciones causales, se basan en planteamientos e hipótesis causales. (Hernández et al., 2014, p. 157)

\subsection{Muestra}

De acuerdo a Hernández et al. (2014), el diseño de la muestra es probabilístico de tipo estratificado. Se optó por clasificar a los estudiantes de la Facultad de Economía de una Universidad Pública de Lima por año académico. De esta población, se procedió a seleccionar una muestra representativa utilizando la técnica de muestreo aleatorio y por afijación proporcional. El tamaño de la muestra se calculó utilizando la fórmula de Sierra Bravo (2001), la que determinó que la muestra estaba compuesta de 318 estudiantes según la siguiente distribución:

Tabla 1

Composición de la muestra por Año Académico

\begin{tabular}{|ccc|}
\hline Año & Frecuencia & Porcentaje \\
\hline I & 60 & 18,9 \\
II & 91 & 28,6 \\
III & 73 & 23,0 \\
IV & 46 & 14,5 \\
V & 48 & 15,1 \\
\hline Total & 318 & 100,0 \\
\hline
\end{tabular}

Fuente: Elaboración Propia 


\subsection{Instrumentos}

En la presente investigación se utilizaron dos instrumentos de recolección de datos:

- Estilos cerebrales de Pensamiento: Se utilizó el Inventario de Estilos Cerebrales de Pensamiento de Carlos Alberto Jiménez.

- Comportamiento del Consumidor: Se utilizó el Inventario del comportamiento del consumidor de Sproles, G.B. y Kendall, E.L., adaptado por el autor de la presente investigación.

\subsection{Análisis de validez y confiabilidad de los instrumentos}

Para garantizar la correcta utilización de los instrumentos de evaluación se hizo necesario efectuar los análisis de validez y confiabilidad respectivos. Los resultados nos muestran que el Inventario de Estilos Cerebrales de Pensamiento alcanza un alfa de Cronbach de 0,87 lo que indica que el instrumento es confiable. Por su parte, el alfa de Cronbach del Inventario del comportamiento del consumidor de 0,76 , por lo que se determina que el instrumento es igualmente confiable. Respecto a los análisis de Validez de los Instrumentos, estos se realizaron utilizando el Análisis Factorial Exploratorio. Los resultados mostraron la presencia de validez de Constructo para ambos casos.

\section{Resultados}

\subsection{Análisis descriptivos}

En la Tabla 2, se presenta el análisis descriptivo de la muestra. Se incluyen los valores mínimos y máximos, la media aritmética, la desviación estándar (D.E.), la asimetría y curtosis. Los resultados indican que las distribuciones de las puntuaciones son ligeramente asimétricas y además presentan una considerable elevación en los valores centrales de las variables Comportamiento del consumidor más emocional, el Estilo Cerebral A y el Estilo Cerebral B, por lo que se hace necesario evaluar el ajuste de las puntuaciones a la distribución normal. 
Tabla 2

Análisis descriptivo de las variables estudiadas

\begin{tabular}{|cccccccc|}
\hline Variables & Mínimo & Máximo & Media & D.E. & Asimetría & Curtosis \\
\hline $\begin{array}{c}\text { Comportamiento del consumidor } \\
\text { más emocional }\end{array}$ & 7,25 & 23,50 & 15,43 & 2,96 & $-0,12$ & 0,01 \\
$\begin{array}{c}\text { Comportamiento del consumidor } \\
\text { más racional }\end{array}$ & 7,67 & 23,00 & 17,83 & 2,31 & $-1,16$ & 3,32 \\
Estilo cerebral A & 24,00 & 98,00 & 73,91 & 10,04 & $-1,31$ & 5,14 \\
Estilo cerebral B & 28,00 & 98,00 & 74,69 & 10,44 & $-1,11$ & 2,89 \\
Estilo cerebral C & 32,00 & 92,00 & 68,75 & 9,66 & $-0,70$ & 0,92 \\
Estilo cerebral D & 28,00 & 98,00 & 71,81 & 9,63 & $-1,06$ & 3,24 \\
\hline
\end{tabular}

Fuente: Elaboración Propia

\subsection{Análisis de la normalidad de las distribuciones de las variables}

Empleando la prueba de Bondad de ajuste a la curva normal de Kolmogorov-Smirnov, se analizaron si las distribuciones de las variables estudiadas se aproximaban a una distribución normal a nivel poblacional. Los resultados presentados en la tabla 3 , muestra que en todos los casos se obtuvieron distribuciones que son estadísticamente significativas, por lo que se concluye que no se aproximaban a la normal a nivel poblacional. Por ello, se decidió normalizar las variables a través de la transformación logarítmica, de manera que se pueden aplicar los contrastes estadísticos paramétricos.

Tabla 3

Pruebas de normalidad Kolmogorov-Smirnov

\begin{tabular}{|cccc|}
\hline Variables & K-S Z & gl & Sig. \\
\hline Comportamiento del consumidor más emocional &, 054 & 318 &, 026 \\
Comportamiento del consumidor más racional &, 106 & 318 &, 000 \\
Estilo cerebral A &, 104 & 318 &, 000 \\
Estilo cerebral B &, 140 & 318 &, 000 \\
Estilo cerebral C &, 102 & 318 &, 000 \\
Estilo cerebral D &, 105 & 318 &, 000 \\
\hline
\end{tabular}

Fuente: Elaboración Propia 


\subsection{Análisis de Regresión}

\subsubsection{Análisis de Regresión Múltiple jerárquica del Comportamiento del consumidor más emocional}

A fin de establecer si los Estilos Cerebrales de Pensamiento podían predecir los puntajes del consumidor más emocional del Comportamiento del Consumidor, se aplicó el análisis de regresión múltiple jerárquica, el cual se desarrolló en dos etapas. En la primera se incluyeron como control sólo las variables sociodemográficas: sexo y edad. En la segunda etapa, se introdujeron los puntajes obtenidos en los cuadrantes como variables predictoras y el Comportamiento del consumidor más emocional como variable predicha.

Los resultados del análisis desarrollado incluido en la tabla 4, permitieron establecer que el modelo 1, el cual incluía las variables de control, presentaron una correlación múltiple baja y que permitía explicar el 2,4\% de la varianza explicada, pero en el modelo 2 , en el cual se incluyen las variables independientes, indica que la correlación múltiple se incrementa a 0,52 que puede clasificarse como moderado y que explica el $7,6 \%$ de la varianza explicada, esto indicaría la presencia de variables predictoras.

Tabla 4

Resumen del modelo de regresión múltiple del Comportamiento del consumidor más emocional

\begin{tabular}{|c|c|c|c|c|c|c|c|}
\hline \multirow[b]{2}{*}{ Modelo } & \multicolumn{5}{|c|}{ Cambio en } & \multirow[b]{2}{*}{$\mathbf{F}$} & \multirow[b]{2}{*}{ sig } \\
\hline & $\mathbf{R}$ & $\mathbf{R}^{2}$ & $\mathbf{R}^{2}$ & gl1 & gl2 & & \\
\hline 1 &, $154^{a}$ & ,024 & ,024 & 2 & 315 & 3,813 &, $023^{\mathrm{b}}$ \\
\hline 2 & $276^{\mathrm{b}}$ & ,076 & 052 & 4 & 311 & 4,261 &, $000^{c}$ \\
\hline
\end{tabular}

Notas: ${ }^{a}$ Variable dependiente: Comportamiento del consumidor más emocional. ${ }^{\mathrm{b}}$ Predictores del modelo 1: (Cons-

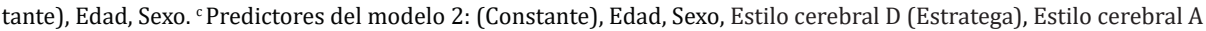
(Experto), Estilo cerebral C (Comunicador), Estilo cerebral B (Organizador)

Fuente: Elaboración Propia

En lo que respecta al análisis de la significación estadística, permite notar que el primer modelo es estadísticamente significativo (F2, gl2 = $315)=3,813 p<, 05$ ), lo cual corrobora que las variables de control producen un pequeño efecto, en tanto que el segundo modelo sí resulta estadísticamente significativo (F4, gl2 $=311)=4,261 \mathrm{p}<, 001)$, lo cual corrobora que las variables predictoras si predicen los valores de la variable Comportamiento del consumidor más emocional. 
Al analizar los resultados específicos de la regresión múltiple jerárquica presentados en la tabla 5 , se encuentra que, en el primer modelo, el sexo presenta un valor que es estadísticamente significativo $(\mathrm{p}<, 01)$. Por otra parte, en el segundo modelo se encuentra que solo los puntajes del Estilo Cerebral C son estadísticamente significativos $(t=2,10 \mathrm{p}<, 05)$, de manera que sería este estilo cerebral el que mejor predice el Comportamiento del consumidor más emocional.

Tabla 5

Coeficientes del análisis de regresión múltiple jerárquica del Comportamiento del consumidor más emocional

\begin{tabular}{|c|c|c|c|c|c|c|c|c|}
\hline & \multirow{2}{*}{ Modelo } & \multicolumn{2}{|c|}{$\begin{array}{l}\text { Coeficientes no } \\
\text { estandarizados }\end{array}$} & \multirow{2}{*}{$\begin{array}{c}\text { Coeficiente } \\
\text { estándar } \\
\text { Beta }\end{array}$} & \multirow{2}{*}{$\mathbf{t}$} & \multirow{2}{*}{ Sig. } & \multicolumn{2}{|c|}{$\begin{array}{c}95 \% \text { intervalo de confi- } \\
\text { anza para B }\end{array}$} \\
\hline & & B & $\begin{array}{c}\text { Error } \\
\text { estándar }\end{array}$ & & & & $\begin{array}{l}\text { Límite } \\
\text { inferior }\end{array}$ & $\begin{array}{l}\text { Límite } \\
\text { superior }\end{array}$ \\
\hline \multirow[t]{3}{*}{1} & (Constante) & 14,116 & 1,305 & & 10,818 & ,000 & 11,548 & 16,683 \\
\hline & Sexo & 914 & ,331 & ,154 & 2,761 & ,006 & ,263 & 1,565 \\
\hline & Edad & ,000 & ,057 & ,000 &,- 004 & ,997 &,- 112 & 112 \\
\hline \multirow[t]{7}{*}{2} & (Constante) & 10,948 & 1,818 & & 6,022 & ,000 & 7,370 & 14,525 \\
\hline & Sexo & 657 & ,335 & 111 & 1,961 & 051 &,- 002 & 1,317 \\
\hline & Edad &,- 032 & ,057 &,- 031 &,- 566 & ,572 &,- 144 & ,080 \\
\hline & Estilo cerebral A &,- 041 & ,025 &,- 139 & $-1,644$ & 101 &,- 090 & ,008 \\
\hline & Estilo cerebral B & ,031 & ,025 & 111 & 1,235 & ,218 &,- 019 & ,082 \\
\hline & Estilo cerebral C & ,051 &, 024 & 165 & 2,101 & ,036 & , 003 & ,098 \\
\hline & Estilo cerebral D & ,020 & ,027 & 064 & 743 & ,458 &,- 033 & ,072 \\
\hline
\end{tabular}

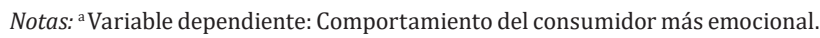

Fuente: Elaboración Propia

4.3.2. Análisis de Regresión Múltiple jerárquica del Comportamiento del consumidor más racional

Al igual que con el consumidor más emocional del Comportamiento del Consumidor, también se realizó el análisis de regresión múltiple jerárquica con el consumidor más racional del Comportamiento del Consumidor como variable predicha. Las etapas son las mismas que en el factor anterior: en una primera etapa se incluyeron solo las variables sociodemográficas(sexo y edad), para luego, en la segunda etapa, incluir los puntajes obtenidos en los cuadrantes como variables predictoras. 
Los resultados del análisis desarrollado incluido en la tabla 6 permitieron establecer que el modelo 1, el cual incluía las variables de control, presentó una correlación múltiple baja y que permitía explicar el $0,4 \%$ de la varianza explicada. Pero en el modelo 2 , en el cual se incluyen las variables independientes, indica que la correlación múltiple se incrementa a 0,60 que puede clasificarse como moderado y que explica el 35,7 \% de la varianza explicada, esto indicaría la presencia de variables predictoras.

Tabla 6

Resumen del modelo de regresión múltiple del Comportamiento del consumidor más racional

\begin{tabular}{|ccccccccc|}
\hline Modelo & $\mathbf{R}$ & $\mathbf{R}^{2}$ & $\mathbf{R}^{2}$ & gl1 & gl2 & F & sig \\
\hline $\mathbf{1}$ &, $063^{\mathrm{a}}$ &, 004 &, 004 & 2 & 315 &, 630 &, $533^{\mathrm{b}}$ \\
$\mathbf{2}$ &, $597^{\mathrm{b}}$ &, 357 &, 353 & 4 & 311 & 28,738 &, $000^{\mathrm{c}}$ \\
\hline
\end{tabular}

Notas: a Variable dependiente: Comportamiento del consumidor más emocional. ${ }^{\mathrm{b}}$ Predictores del modelo 1: (Constante), Edad, Sexo. ' Predictores del modelo 2: (Constante), Edad, Sexo, Estilo cerebral D (Estratega), Estilo cerebral A (Experto), Estilo cerebral C (Comunicador), Estilo cerebral B (Organizador) Fuente: Elaboración Propia

El análisis de la significación estadística específica permite notar que el primer modelo no es estadísticamente significativo $(\mathrm{F} 2, \mathrm{gl} 2=315)=$ $0,630 p>, 533$ ), lo cual corrobora que las variables de control no producen efecto alguno, en tanto que el segundo modelo si resulta estadísticamente significativo $(\mathrm{F} 4, \mathrm{gl} 2=311)=28,738 \mathrm{p}<, 001)$, lo cual corrobora que las variables predictoras sí predicen los valores de la variable Comportamiento del consumidor más racional.

El análisis de los resultados específicos de la regresión múltiple jerárquica presentados en la tabla 7 , permite apreciar que, en el primer modelo, el sexo y la edad presentan valores que no son estadísticamente significativos $(p>, 05)$. En tanto que en el segundo modelo se encuentra que tanto los puntajes del estilo cerebral B $(\mathrm{t}=3,634 \mathrm{p}<, 001)$ y del estilo cerebral $\mathrm{D}(\mathrm{t}=2,943 \mathrm{p}<, 01)$ son estadísticamente significativos, de manera que serían estos dos cuadrantes los que mejor predicen los puntajes del Comportamiento del consumidor más racional. 
Tabla 7

Coeficientes del análisis de regresión múltiple jerárquica del Comportamiento del consumidor más racional

\begin{tabular}{|c|c|c|c|c|c|c|c|c|}
\hline & \multirow{2}{*}{ Modelo } & \multicolumn{2}{|c|}{$\begin{array}{l}\text { Coeficientes no } \\
\text { estandarizados }\end{array}$} & \multirow{2}{*}{$\begin{array}{c}\begin{array}{c}\text { Coeficiente } \\
\text { estándar }\end{array} \\
\text { Beta }\end{array}$} & \multirow{2}{*}{$\mathbf{t}$} & \multirow{2}{*}{ Sig. } & \multicolumn{2}{|c|}{$\begin{array}{l}95 \% \text { intervalo de } \\
\text { confianza para B }\end{array}$} \\
\hline & & B & $\begin{array}{c}\text { Error } \\
\text { estándar }\end{array}$ & & & & $\begin{array}{l}\text { Límite } \\
\text { inferior }\end{array}$ & $\begin{array}{c}\text { Límite } \\
\text { superior }\end{array}$ \\
\hline \multirow[t]{3}{*}{1} & (Constante) & 18,140 & 1,030 & & 17,614 & ,001 & 16,113 & 20,166 \\
\hline & Sexo &,- 291 & 261 &,- 063 & $-1,114$ & ,266 &,- 805 & ,223 \\
\hline & Edad & ,005 & ,045 & ,007 & 119 & 906 &,- 083 & ,094 \\
\hline \multirow[t]{7}{*}{2} & (Constante) & 7,717 & 1,185 & & 6,510 & ,001 & 5,385 & 10,050 \\
\hline & Sexo &,- 372 & ,219 &,- 080 & $-1,704$ & , 089 &,- 803 & ,058 \\
\hline & Edad &,- 045 & ,037 &,- 056 & $-1,206$ & ,229 &,- 118 & ,028 \\
\hline & Estilo cerebral A & ,025 & ,016 & 107 & 1,509 & ,132 &,- 007 &, 057 \\
\hline & Estilo cerebral B & ,060 & ,017 & ,273 & 3,634 & ,001 & ,028 & ,093 \\
\hline & Estilo cerebral C & ,023 & ,016 & ,098 & 1,493 & ,136 &,- 007 &, 054 \\
\hline & Estilo cerebral D & 051 & 017 & ,212 & 2,943 & ,003 & ,017 & 085 \\
\hline
\end{tabular}

Nota: Variable dependiente: consumidor más racional.

Fuente: Elaboración Propia

\subsection{Análisis de ecuaciones estructurales a través del programa AMOS}

A fin analizar la estructura de las relaciones complejas entre variables, se aplicó el análisis de senderos (path analysis) con el programa IBM-SPSS AMOS v24, asumiendo los Factores 1 y 2 (consumidor más emocional y racional), del Comportamiento del consumidor como variables dependientes y a los cuatro estilos cerebrales de pensamiento como variables independientes. Los resultados incluidos en la tabla 8 y el gráfico № 2indican que las variables que influyen de forma estadística significativa sobre los puntajes del Comportamiento del consumidor más emocional son el Estilo Cerebral A $(\mathrm{RC}=-2.072 \mathrm{p}<.05)$ y el Estilo Cerebral C $(\mathrm{RC}=2.278 \mathrm{p}$ $<.05)$, debiendo notarse que el efecto se da de forma inversa en el cuadro del estilo cerebral A. En tanto que en los puntajes del Comportamiento del consumidor más racional, las variables que la influyen son los puntajes del Estilo Cerebral B ( RC $=3.518 \mathrm{p}<.05)$ y el Estilo Cerebral D ( $\mathrm{RC}=2.950$ $\mathrm{p}<.01$ ), debiendo notarse que en este caso los efectos son positivos.

El análisis de la significación del modelo indica que se obtiene una Chicuadrada que es estadísticamente significativa (Chi-Cuadrado(15)=31.08 p < $.05)$ lo cual podría deberse al tamaño de la muestra $(n=318)$, en tanto que en 
los coeficientes que corresponden al promedio de la raíz cuadrada de los residuales (RMR = 0.06), y el índice de bondad de ajuste del modelo (GFI = 0.99), presentan resultados que permiten concluir que modelo es adecuado y garantiza que constituye una buena solución para las ecuaciones estructurales.

Tabla 8

Análisis de la estimación de los parámetros del modelo de ecuaciones estructurales de los factores del Comportamiento del consumidor

\begin{tabular}{|cccccccc|}
\hline V. I. & & V. D. & $\begin{array}{c}\text { Valor } \\
\text { estimado }\end{array}$ & $\begin{array}{c}\text { Valor estimado } \\
\text { estand. }\end{array}$ & S.E. & $\begin{array}{c}\text { Razón } \\
\text { crítica. }\end{array}$ & Sig. \\
\hline Cuadrante A & $---\rightarrow$ & CSIF1 & -.051 &,- 17 & -2.072 & -2.072 & $.038^{*}$ \\
Cuadrante B & $---\rightarrow$ & CSIF1 & .031 &, 11 & 1.216 & 1.216 & .224 \\
Cuadrante C & $---\rightarrow$ & CSIF1 & .054 &, 18 & 2.278 & 2.278 & $.023^{*}$ \\
Cuadrante D & $---\rightarrow$ & CSIF1 & .027 &, 09 & 1.016 & 1.016 & .310 \\
Cuadrante A & $---\rightarrow$ & CSIF2 & .030 &, 13 & 1.896 & 1.896 & .058 \\
Cuadrante B & $---\rightarrow$ & CSIF2 & .058 &, 26 & 3.518 & 3.518 & $.001^{* * *}$ \\
Cuadrante C & $---\rightarrow$ & CSIF2 & .018 &, 07 & 1.196 & 1.196 & .232 \\
Cuadrante D & $---\rightarrow$ & CSIF2 & .050 &, 21 & 2.950 & 2.950 & $.003^{* *}$ \\
\hline
\end{tabular}

CSIF1 $=$ Comportamiento del consumidor más emocional

CSIF2 = Comportamiento del consumidor más racional

Fuente: Elaboración Propia

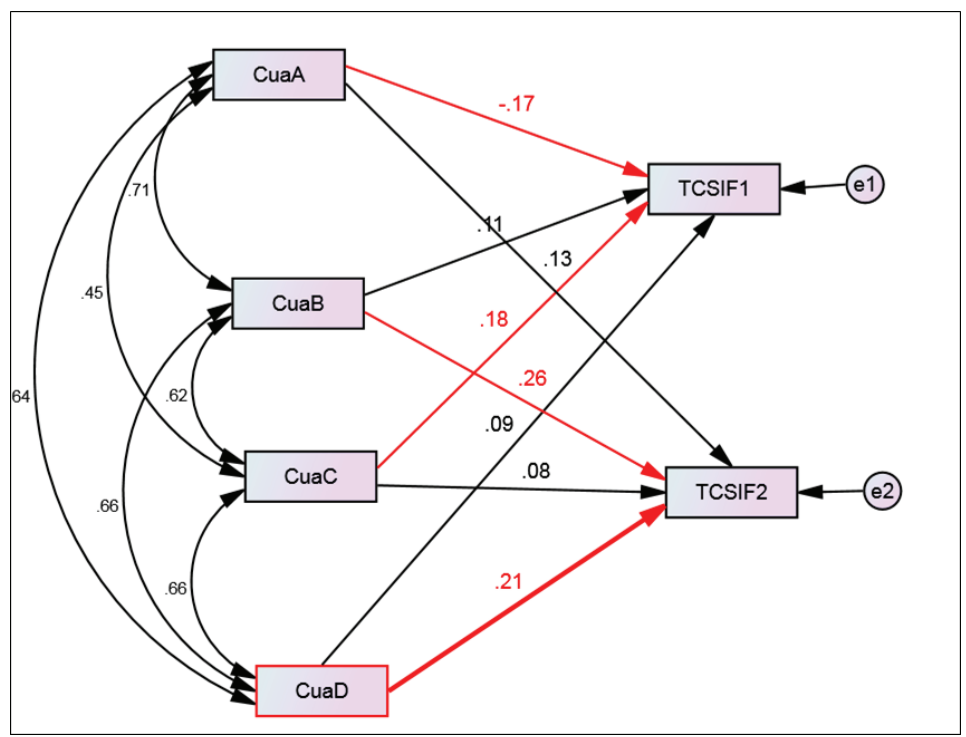

Gráfico 2. Modelo de influencia de los cuadrantes sobre los Factores del Comportamiento del Consumidor

Fuente: Elaboración Propia 


\subsection{Análisis correlacional}

Tabla 9

Correlaciones entre los Estilos Cerebrales de Pensamiento y el Comportamiento del consumidor más emocional

\begin{tabular}{|lc|}
\hline \multicolumn{1}{|c|}{ Variables } & Comportamiento del consumidor más emocional \\
\hline Estilo cerebral A (Experto) & 0,040 \\
Estilo cerebral B (Organizador) & $0,152^{* *}$ \\
Estilo cerebral C (Comunicador) & $0,224^{* *}$ \\
Estilo cerebral D (Estratega) & $0,165^{* *}$ \\
\hline
\end{tabular}

${ }^{*} p<, 05{ }^{* *} p<, 01{ }^{* * *} p<, 001$

Fuente: Elaboración Propia

El análisis de las correlaciones de efectuados con la $\mathrm{r}$ de Pearson presentadas en la tabla 9, permite apreciar que en el caso del Comportamiento del consumidor más emocional se encuentran correlaciones positivas, estadísticamente significativas pero bajas con el estilo cerebral B $(\mathrm{r}=0,152 \mathrm{p}<.01)$, con el estilo cerebral $\mathrm{C}(\mathrm{r}=0,224 \mathrm{p}<.01)$ y con el estilo cerebral D $(r=0,165 \mathrm{p}<.01)$.

Tabla 10

Correlaciones entre los Estilos Cerebrales de Pensamiento y el Comportamiento del consumidor más racional

\begin{tabular}{|lc|}
\hline \multicolumn{1}{|c|}{ Variables } & Comportamiento del consumidor más racional \\
\hline Estilo cerebral A (Experto) & $0,487^{* * *}$ \\
Estilo cerebral B (Organizador) & $0,541^{* * *}$ \\
Estilo cerebral C (Comunicador) & $0,437^{* * *}$ \\
Estilo cerebral D (Estratega) & $0,517^{* * *}$ \\
\hline
\end{tabular}

${ }^{*} p<, 05 * * p<, 01 * * * p<, 001$

Fuente: Elaboración Propia

El análisis de las correlaciones de efectuados con la $\mathrm{r}$ de Pearson presentadas en la tabla 10, permite apreciar que el en el caso del Comportamiento del consumidor más racional se observan correlaciones estadísticamente significativas y positivas con cada uno de los cuadrantes, notándose que las correlaciones son fuertes con el estilo cerebral B ( $\mathrm{r}=$ $0,541 \mathrm{p}<.001)$ y el estilo cerebral $\mathrm{D}(\mathrm{r}=0,517 \mathrm{p}<.001)$, y son clasificadas como moderadas con el estilo cerebral A $(r=0,487 \mathrm{p}<.01)$ y con el estilo cerebral C $(\mathrm{r}=0,437 \mathrm{p}<.01)$. 


\subsection{Discusión de los resultados}

Los resultados obtenidos y expresados en las tablas 5 y 7 nos muestran que sí existe influencia de los Estilos Cerebrales de Pensamiento sobre el Comportamiento del Consumidor en los estudiantes, por lo que se puede afirmar que la primera hipótesis de investigación ha sido confirmada. Los hallazgos se confirman con el análisis de ecuaciones estructurales (Tabla 8). Esta confirmación va en línea con lo mencionado en la introducción del presente artículo: la toma de decisiones humanas (para efectos de esta investigación, la toma de decisiones de compra) no necesariamente sigue un proceso racional, lo cual hace fundamental conocer las áreas del cerebro que se activan al momento de decidir la compra. El Modelo del Cerebro Total de Herrmann (1976) es una herramienta importante para determinar el estilo cerebral de pensamiento predominante en el consumidor. Además, se puede complementar con el uso de herramientas tecnológicas como el eye-tracker(técnica de seguimiento de ojos) o escáneres de resonancia magnética para dar respuestas aún más certeras.

En cuanto a las siguientes hipótesis, se tiene que sí existen relaciones significativas entre los Estilos Cerebrales de Pensamiento y el Comportamiento del Consumidor más Emocional, compuesto por las siguientes dimensiones: Consumidor Recreacional, de Moda, de Marca y Leales a una marca. En este caso, las relaciones significativas existentes son positivas con los estilos B (Organizador), C (Comunicador) y D (Estratega) según lo expresado en la tabla 9.

Para el tercer caso, sí hay relaciones significativas entre los Estilos Cerebrales de Pensamiento y el Comportamiento del Consumidor más Racional, cuyas dimensiones son: Consumidores Perfeccionistas, Conscientes del Precio y Confundidos. Los estilos este caso, las relaciones significativas existentes son positivas con los estilos B (Organizador) y D (Estratega) tienen una correlación positiva y fuerte, mientras que los estilos A (Experto) y C (Comunicador), tienen una correlación positiva y moderada, tal como se expresa en la tabla 10. La activación del estilo C nos muestra que, aún cuando la racionalidad es más fuerte en este tipo de consumidores, la emoción está presente y forma parte del proceso de toma de decisiones. 
Así, podemos ver que los Estilos Cerebrales de Pensamiento tienen influencia sobre los dos factores que explican el Comportamiento del Consumidor, y que su influencia varía dependiendo si el consumidor es más emocional o más racional.

En cuanto a la predicción, los resultados del análisis de regresión múltiple jerárquica muestran que sí se puede predecir la presencia de un comportamiento determinado del consumidor. Ese tipo de análisis se realizó para encontrar el o los estilos cerebrales que mejor predicen un determinado factor. Así, para el caso del consumidor más emocional, el estilo cerebral de pensamiento que mejor lo predice es el C (Comunicador), tal como se establece en la tabla 5. De esa manera, podemos afirmar que el factor 1 del Comportamiento del Consumidor es un Factor Emocional, por lo que el estudiante que tenga una de las dimensiones que lo compone, tiende a decidir qué comprar por impulsos emocionales. Por ello, la tendencia de este tipo de consumidores es a tener el tipo de conducta que Ariely (2008) sostiene: son irracionales (compran algo que no necesitan pero que les gustó o está a la moda y por eso lo quieren) y toman ese tipo de decisiones con tal frecuencia que se torna predecible. Que una empresa tenga en su poder este tipo de información puede acarrear problemas éticos si no es utilizada adecuadamente (por ejemplo, si se pretende usar para forzar compras de una manera desmedida).

Mientras que, para el caso del consumidor más racional, son los estilos B (Organizador) y D, tal como se menciona en la tabla 7. Así, podemos afirmar que el factor 2 del Comportamiento del Consumidor es un Factor Racional, pues se relaciona directamente con el estilo B (Organizador) y el D (Estratega). Las dimensiones que incorporan este factor están relacionadas a estilos en los que el consumidor razona, se organiza y se informa antes de decidir su compra, por lo que estos estudiantes son conscientes de lo que buscan y de las restricciones (presupuesto, tiempo, etc.) que puedan tener. Al tener activo el estilo D (Estratega), una de las maneras en las que este tipo de consumidor se informa es a través de las relaciones sociales. Así, puede pedir consejos y/o recomendaciones a sus familiares, amigos y otras personas con las que se relacione. Como afirman Kotler y Armstrong (2012), los factores sociales influyen al momento de decidir una compra y en una persona con un estilo D (Estratega), eso queda claramente de manifiesto. 
Los resultados anteriores nos permiten establecer un vínculo con los sistemas de pensamiento que Kahneman (2011, p.35) denomina "Sistema 1" y "Sistema 2", también conocidos como "Sistema Automático" y "Sistema Reflexivo" (Thaler y Sunstein, 2008, p.22). El consumidor más emocional tiene elementos del Sistema 1, pues decide rápido, de manera automática en muchos casos, y sin mayor esfuerzo. "Es mi marca de toda la vida y no la cambio por nada", puede pensar un consumidor leal a una marca. Por otra parte, el consumidor más racional muestra elementos del Sistema 2, pues sus decisiones requieren esfuerzo a nivel cerebral antes de decidir. Un consumidor perfeccionista, como alguien que está buscando comprar un auto, se informará bien y comparará opciones antes de tomar una decisión. Ese esfuerzo no es tan automático y requiere tiempo.

Los resultados del análisis del modelo de ecuaciones estructurales (tabla 8) muestran claramente este análisis. En el consumidor más emocional, la activación (negativa) del estilo A, el más racional, es solo para mostrar que cede el control de la toma de decisiones al estilo $\mathrm{C}$, que es el más emocional. Pero el estilo $\mathrm{C}$ también está activo en los consumidores más racionales, y puede jugar un rol importante en la toma de decisiones, particularmente en situaciones de alto estrés o en un consumidor confundido por las opciones. Esto va en línea con los planteamientos de Kahneman (2011), quien afirma que cuando el Sistema 2 entra en fase de agotamiento, el Sistema 1 toma el control y decide.

Es preciso mencionar que la variable de control sexo sí produce un pequeño efecto sobre el Factor Emocional, lo que nos permite sugerir la realización de mayores estudios sobre esta característica. De esa manera, se podría determinar si se trata de una característica propia del segmento estudiado en esta investigación (estudiantes universitarios) o si es una característica extrapolable a diversos grupos poblacionales. Según Kotler y Armstrong (2012), los factores personales, como la variable sexo, sí tienen influencia sobre la decisión que se tomará. Podríamos argumentar a priori que esto se debe a que la mujer es un ser más emocional según algunas investigaciones psicológicas, pero puede que esto esté cambiando, de ahí la sugerencia de ahondar en la investigación de esta variable. 


\section{Reflexiones y conclusiones}

A nivel empresarial, conocer estos resultados permite a las empresas un mejor entendimiento de un grupo de consumidores potencial o actual, lo cual devendrá en un diseño de productos y estrategias acorde con ello para captar más consumidores del segmento estudiantil (en su mayoría jóvenes) y mantener los actuales. En esa línea, por ejemplo, se podrán crear estrategias de fidelización de clientes con un componente emocional alto dirigido a estudiantes que tengan un estilo $\mathrm{C}$ bastante elevado. Para estudiantes con estilos más racionales, como el B, se pueden diseñar productos y servicios teniendo en mente las determinadas cualidades que busca en un producto que será comparado con otros (por ejemplo, un precio bajo y una buena calidad del producto pueden otorgar una ventaja determinante a un bien en comparación con otro si el estudiante compara en precios). Aunque la aplicación de estas técnicas puede representar inicialmente una inversión importante para las empresas, una adecuada aplicación puede generar un impacto positivo en las utilidades de la empresa, puesto que dispondrán de información altamente específica sobre sus potenciales clientes, por lo que los productos que lanzarán al mercado y las campañas de marketing estarán dirigidos a satisfacer necesidades exactas de un sector de clientes que, al ver que el producto cumple con todos sus requerimientos, lo comprarán. De esta forma, las empresas captarán un número mayor de clientes, obteniendo ventas mayores que repercuten en sus utilidades. Organismos gubernamentales e internacionales también pueden emplear este tipo de resultados para plantear campañas de políticas públicas. Como se ha mencionado anteriormente, el uso de herramientas de la neuroeconomía permitirá obtener resultados aún más exactos, puesto que permitirá conocer con certeza las zonas del cerebro que se activan cuando se enfrentan a los diferentes estímulos que se encuentran en, para efectos del ejemplo anterior, un local comercial.

A nivel académico, los resultados permiten a la universidad conocer cómo deciden sus alumnos qué comprar. Así, ellos pueden ofrecer orientación sobre el cuidado del dinero (para consumidores impulsivos, por ejemplo) o servicios orientados a satisfacer necesidades específicas (como cursos de especialización). Al ser este un campo de estudio joven, desde 
la universidad se debería ofrecer cursos sobre este campo para alentar el estudio y estimular el debate académico sobre los potenciales beneficios y dificultades de su aplicación.

\section{Referencias}

Ariely, D. (2008). Predictably irrational. Harper Collins. Estados Unidos.

Ariely, D., Loewenstein, G., \& Prelec, D. (2006). Tom Sawyer and the construction of value. Journal of Economic Behavior \& Organization, 60, 1-10.

Herrmann International. (2005). Ned Herrmann: Pioneer of creative thinking in the corporation and founder of Whole Brain Technology. Recuperado el 15 de junio de 2016, de Herrmann International: https://web.archive.org/ web/20050306232953/http://www.hbdi.com/ned_herrmann.htm

Herrmann International. (2009). The Theory Behind the HBDI® and Whole Brain Technology.Lake Lure: Herrmann International.

Herrmann International Latin America. (2004). Tecnología Herrmann de Dominancia Cerebral - Aplicaciones de Éxito. Recuperado el 16 de mayo de 2017, de Herrmann International Latin America: http://herrmannlatin.com/aplicacion.php

Herrmann Solutions. (2015). Client Success Stories. Recuperado el 6 de setiembre de 2017, de Herrmann International: http://www.herrmannsolutions.com/ results/

Herrmann Solutions. (2016). What is Whole Brain ${ }^{\circledR}$ Thinking?Recuperado el 21 de noviembre de 2016, de Herrmann International: https://www.herrmannsolutions.com/what-is-whole-brain-thinking-2/

Herrmann, N., \& Herrmann-Nehdi, A. (2015). The Whole Brain Business Book (2o ed.). McGraw Hill Education.

Hernández, R., Fernández, C. y Baptista, P. (2014). Metodología de la Investigación. México. Ed McGraw Hill.

Hoyer, W. D., MacInnis, D. J., \& Pieters, R. (2018). Consumer Behaviour (7 ed.). Boston, MA: Cengage Learning.

Jiménez, C. A. (s.f.). Prueba de Diagnóstico sobre Predominancia Cerebral de Consumo de acuerdo a los Estilos Cerebrales de Pensamiento. Recuperado el 16 de junio de 2016, de Neuromarketing - Dr. Carlos Alberto Jiménez Vélez: http:// www.neuromarketing.com.co/diagnosticos_neuromarketing.html 
Jiménez, C. A. (s.f.). Teoría del Cerebro Total. Recuperado el 15 de mayo de 2016, de Neuromarketing - Dr. Carlos Alberto Jiménez Vélez: http://www.neuromarketing.com.co/teorias_cerebro_total.html

Kahneman, D. (2011). Pensar rápido, pensar despacio (7ํe․). Barcelona: Debate.

Kahneman, D., \& Tversky, A. (marzo de 1979). Prospect Theory: An analysis of decision uinder risk. Econometrica, 47(2), 263-291.

Kerlinger, F. N. y Lee, H. B. (2002). Investigación del comportamiento. Métodos de investigación en ciencias sociales ( $4^{\underline{a}}$ ed.). México: McGraw-Hill.

Kotler, P. y Armstrong, G. (2012). Marketing. Ed. Pearson Educación. México.

Nobel Media. (2002). Daniel Kahneman - Facts . Recuperado el 7 de septiembre de 2017, de Nobelprize.org: https://www.nobelprize.org/nobel_prizes/economic-sciences/laureates/2002/kahneman-facts.html

Pico, I. (2016). La dominancia cerebral según los cuadrantes de Herrmann. Recuperado el 15 de mayo de 2017, de PsicoPico: http://psicopico.com/cuadrantes-cerebrales-de-herrman/

Sanchez, H., \& Reyes, C. (2006). Metodología y Diseños en la Investigación Científica. Lima: Editorial Universidad Ricardo Palma.

Schiffman, L. G., \&Kanuk, L. L. (2010). Comportamiento del consumidor (10ํed.). Ciudad de México: Pearson Educación.

Sierra Bravo, R. (2001). Técnicas de investigación social: Teoría y ejercicios. Madrid. Editorial Paraninfo.

Sivakumar, J., \& Kamaraj, M. (noviembre de 2014). Decision-making behaviour towards electronic goods: a study of male and female consumers in Chennai City. Recuperado el 21 de agosto de 2017, de International Journal of Management and Social Science Research Review: http://ijmsrr.com/ downloads/30112014IJMSRR\%207.pdf

Sproles, G. B., \& Kendall, E. L. (diciembre de 1986). A Methodology for Profiling Consumers' Decision-Making Styles. Journal of Consumer Affairs, 20(2), 267269.

Thaler, R., \& Sunstein, C. (2008). Nudge: Improving decisions about health, wealth and happiness. London: Penguin Books.

TorresLajo, M. (2017). Estilos Cerebrales de Pensamiento Vinculados al Comportamiento del Consumidor en Estudiantes Universitarios. Tesis para optar al Título Profesional de Economía. Facultad de Ciencias Económicas. UNMSM. 
Zorrilla, S. (2007). Introducción a la metodología de la investigación. Ciudad de México: Aguilar y León, Cal Editores.

\section{Notas al final}

1 El presente artículo es una síntesis de la Tesis del mismo nombre, que fue presentada para optar al Título Profesional de Economista y aprobada por unanimidad en la Facultad de Ciencias Económicas de la Universidad Nacional Mayor de San Marcos (2017).

2 Economista por la Universidad Nacional Mayor de San Marcos (Perú). Estudiante de Maestría de la Technische Universität München, School of Management (Alemania).E-mail: manuel.torres-lajo@tum.de; mptorreslajo@me.com. 